\title{
Functionalized nanocelluloses as bio-based chemicals in froth flotation
}

\author{
Feliciana Ludovici, *, Ana Luiza C.B. de Carvalho², Robert Hartmann ${ }^{3}$, Henrikki Liimatainen ${ }^{1}$ \\ ${ }^{1}$ Fiber and Particle Engineering Research Unit, University of Oulu, P. O. Box 4300, FI-90014 Oulu, Finland \\ 2Department of Mineral and Waste Processing, Institute of Mineral and Waste Processing, Waste Disposal \\ and Geomechanics, Technical University of Clausthal, Clausthal-Zellerfeld, Germany \\ ${ }^{3}$ Department of Chemical and Metallurgical Engineering, Aalto University, P.O. Box 11000, Fl-00076 Espoo, \\ Finland \\ *Corresponding author: Tel +358 504488072, E-mail: feliciana.ludovici@oulu.fi
}

Froth flotation is the most common process in the mining industry for the beneficiation of complex sulfide ores. For this purpose, thiol collectors (mostly xanthates) are typically used as flotation chemicals because of their efficacy. However, concerns for their effect on human health and negative impact on the environment increasingly urge for alternative candidates. Consequently, there are great ecological and economical interests to develop novel green chemicals from renewable resources to overcome the inherent environmental and health problems associated with traditional petroleum-derived mining chemicals.

Cellulose, which is the most abundant natural polymer resource, represents a green alternative raw material to develop sustainable chemicals that could replace currently used synthetic additives. In the present work, we introduce a novel environmentally friendly and industrially feasible process to produce selective froth flotation chemicals. Therefore, the pulp fibers were disintegrated to cellulose nanoparticles and further functionalized with a silylation reaction in aqueous conditions. Two different functional groups were incorporated into the cellulose nanoparticles and subsequently investigated, namely a thiol-functional moiety, which has an affinity towards pyrite ore surfaces, or an amine-functional moiety, which has an affinity towards silica surfaces. Microflotation tests were carried out in a Hallimond tube to study the flotability of pure pyrite or quartz as a function of $\mathrm{pH}$ value, collector concentration, flotation time, and gas flow rate using the novel green nanoparticles, and their results were compared with commercial xanthate and amine chemicals.

Keywords: cellulose nanoparticles, deep-eutectic solvent, silylation; ore beneficiation, froth flotation. 